\title{
The Star Formation Rate-Dense Gas Relation in the Nuclei of Nearby Galaxies
}

\section{Citation}

Narayanan, Desika, Thomas J. Cox, and Lars Hernquist. 2008. “The Star Formation Rate-Dense Gas Relation in the Nuclei of Nearby Galaxies." The Astrophysical Journal 681 (2): L77-80. https://doi.org/10.1086/590342.

\section{Permanent link}

http://nrs.harvard.edu/urn-3:HUL.InstRepos:41381685

\section{Terms of Use}

This article was downloaded from Harvard University's DASH repository, and is made available under the terms and conditions applicable to Open Access Policy Articles, as set forth at http:// nrs.harvard.edu/urn-3:HUL.InstRepos:dash.current.terms-of-use\#OAP

\section{Share Your Story}

The Harvard community has made this article openly available.

Please share how this access benefits you. Submit a story. 
APJL - ACCEPTED

Preprint typeset using $\mathrm{LT}_{\mathrm{E}} \mathrm{X}$ style emulateapj v. 12/14/05

\title{
THE STAR FORMATION RATE - DENSE GAS RELATION IN THE NUCLEI OF NEARBY GALAXIES
}

\author{
DESIKA NARAYANAN ${ }^{1,2}$, ThOMAS J. COX ${ }^{1,3}$, AND LARS HERnQUIST ${ }^{1}$ \\ ApJL - accepted
}

\begin{abstract}
We investigate the relationship between the star formation rate (SFR) and dense molecular gas mass in the nuclei of galaxies. To do this, we utilize the observed $850 \mu \mathrm{m}$ luminosity as a proxy for the infrared luminosity $\left(\mathrm{L}_{\mathrm{IR}}\right)$ and SFR, and correlate this with the observed $\mathrm{CO}(\mathrm{J}=3-2)$ luminosity. We find tentative evidence that the $\mathrm{L}_{\mathrm{IR}}-\mathrm{CO}(\mathrm{J}=3-2)$ index is similar to the Kennicutt-Schmidt $(\mathrm{KS})$ index $(N \approx 1.5)$ in the central $\sim 1.7 \mathrm{kpc}$ of galaxies, and flattens to a roughly linear index when including emission from the entire galaxy. This result may imply that the volumetric Schmidt relation is the underlying driver behind the observed SFR-dense gas correlations, and provides tentative confirmation for recent numerical models. While the data exclude the possibility of a constant $\mathrm{L}_{\mathrm{IR}}-\mathrm{CO}(\mathrm{J}=3-2)$ index for both galaxy nuclei and global measurements at the $\sim 80 \%$ confidence level, the considerable error bars cannot preclude alternative interpretations.
\end{abstract}

Subject headings: stars: formation - galaxies: ISM, evolution, starburst - radio lines: ISM, galaxies

\section{INTRODUCTION}

The rate at which stars form in galaxies has long been parameterized in terms of a power-law involving the gas density (Schmidt 1959). HI and CO observations of galaxies have shown that the star formation rate (SFR) is related to the surface gas density via $\Sigma_{\text {SFR }} \propto \Sigma_{\text {gas }}^{1.4-1.5}$ (Kennicutt 1998). Theoretically, a power-law index of $\sim 1.5$ controlling the SFR is appealing. If a constant fraction of gas is converted into stars over a free fall time, the relation SFR $\propto \rho_{\text {gas }}^{1.5}$ results.

Observations of molecular gas in galaxies have provided a more complex view of SFR relations. Local surveys have shown that the SFR (as traced by the $\mathrm{L}_{\mathrm{IR}}$ ) is proportional to the CO $(\mathrm{J}=1-0)$ luminosity $\left(L^{\prime}\right.$; Gao \& Solomon 2004a) to the $\sim 1.5$ power, consistent with observed surface density SFR relations (Kennicutt 1998). However, recent observations have revealed a tight, linear relation between $\operatorname{HCN}(\mathrm{J}=1-0)$ and the $\mathrm{L}_{\mathrm{IR}}$ in galaxies (Gao \& Solomon 2004a b). Because the $\mathrm{J}=1$ 0 rotational transition of $\mathrm{HCN}$ has a relatively high critical density $\left(n_{\text {crit }} \sim 10^{5} \mathrm{~cm}^{-3}\right.$ ), this has been interpreted as a more fundamental SFR relation such that the volumetric SFR is linearly related to the dense, star-forming molecular gas. A linear relation between $\mathrm{L}_{\mathrm{IR}}$ and the luminosity from a high critical density tracer $\mathrm{CO}(\mathrm{J}=3-2)$ in a similar sample of galaxies has provided confirming evidence for this relation and advocated a similar interpretation (Narayanan et al. 2005).

An alternative interpretation to the linear relation between $\mathrm{L}_{\mathrm{IR}}$ and dense gas tracers has been put forth from the theoretical side. First, Krumholz \& Thompson (2007) utilized models of individual giant molecular clouds (GMCs) with a lognormal density distribution function coupled with escape probability radiative transfer calculations. These authors found that the central issue driving the observed molecular SFR relations was the relationship between the fraction of the cloud's gas above the critical density of the molecular transition. When, on average, the gas density is higher than the line's critical density $\left(\left\langle n_{\text {cloud }}\right\rangle » n_{\text {crit }}\right)$, the bulk of the gas is thermalized in the line, and the line luminosity increases linearly with increasing mean gas density $(\langle n\rangle)$. In this scenario,

\footnotetext{
${ }^{1}$ Harvard-Smithsonian Center for Astrophysics, 60 Garden Street, Cambridge, MA 02138, USA

2 dnarayanan@cfa.harvard.edu

${ }^{3}$ W.M. Keck Postdoctoral Fellow
}

a relationship between $S F R$ and $L_{\text {mol }}$ is expected to have index similar to the underlying (volumetric) Schmidt index. This is reminiscent of the observed relation between $\mathrm{L}_{\mathrm{IR}}$ and $\mathrm{CO}$ $(\mathrm{J}=1-0)$ in galaxies. Alternatively, when only a small fraction of the gas is thermalized $\left(\left\langle n_{\text {cloud }}\right\rangle\left\langle n_{\text {crit }}\right)\right.$, the line luminosity will increase superlinearly with $\langle n\rangle$, and the SFR-L $\mathrm{L}_{\text {mol }}$ relation will have index less than that of the underlying Schmidt index (e.g. the observed $\mathrm{L}_{\mathrm{IR}}-\mathrm{HCN} \mathrm{J}=1-0$ relation).

Narayanan et al. (2007) furthered these models of GMCs by applying 3D non-LTE radiative transfer calculations to hydrodynamic simulations of isolated galaxies and equal mass binary galaxy mergers. A key finding in these numerical models was that the observed relations are only found when global measurements are made. Higher spatial resolution observations of e.g. the nuclei of galaxies would probe gas in which a larger fraction of the gas is thermalized than in unresolved observations of the entire galaxy. In this case, $\langle n\rangle$ could potentially become comparable to the critical density of the line, and an SFR- $\mathrm{L}_{\text {mol }}$ index of $\sim 1.5$ would be expected even for high critical density molecular lines. In both sets of models, the fundamental relation is the Schmidt relation with index 1.5. The observed SFR- $\mathrm{L}_{\text {mol }}$ relations were simply manifestations of the underlying Schmidt law.

A key difference exists between the numerical models of Krumholz \& Thompson (2007) and Naravanan et al. (2007), and the interpretations of Gao \& Solomon (2004a b), Narayanan et al. (2005) and Wu et al. (2005). The models find the driving relation is the volumetric Schmidt relation whereas the latter set of observations cite a more fundamental relation as that between SFR and dense molecular gas. Tests that distinguish between these interpretations are crucial to understanding global SFR relations in galaxies. Narayanan et al. (2007) offered a direct prediction from their models that observations of molecular lines with critical density higher than that of $\mathrm{HCN}(\mathrm{J}=1-0)$ should break the linear trend seen between SFR and HCN $(\mathrm{J}=1-0)$. Specifically, for extremely high critical density lines, $n_{\text {crit }}$ will be even larger than $\left\langle n_{\text {galaxy }}\right\rangle$ than in the case of $\mathrm{HCN}(\mathrm{J}=1-0)$; In these cases, the relation between $\mathrm{SFR}$ and $\mathrm{L}_{\text {mol }}$ is directly predicted to be sublinear. Indeed, confirming evidence for this trend has been found by Bussmann et al. (2008; submitted), who found a sublinear $\mathrm{L}_{\mathrm{IR}}-\mathrm{HCN}(\mathrm{J}=3-2)$ index in remarkable agreement with the predictions of Narayanan et al. (2007). 
An alternative generic feature of both the Krumholz \& Thompson (2007) and Narayanan et al. (2007) simulations which may serve as a test of the models is a superlinear SFR- $\mathrm{L}_{\text {mol }}$ relation for high critical density tracers when $\left\langle n_{\text {galaxy }}\right\rangle$ is sufficiently high. One potential manifestation of this is a break in the linear SFR-HCN (J=1-0) relation in systems with extremely high infrared luminosity (e.g. hyper-luminous infrared galaxies; $\mathrm{L}_{\mathrm{IR}}>10^{13} \mathrm{~L}_{\odot}$ ). Here, the models predict a steepening in the $S F R-L_{m o l}$ index toward $N=1.5$ (Krumholz \& Thompson 2007). Tentative evidence for this may have been found by Gao et al. (2007) in high redshift systems, though the potential contribution of active galactic nuclei (AGN) to the $\mathrm{L}_{\mathrm{IR}}$ may drive a similar signature.

In order to avoid the muddying effects of central AGN, a potential alternative to this test is to observe the SFR- $\mathrm{L}_{\text {mol }}$ relation in galactic nuclei, where the local mean gas density may be higher than the globally averaged mean gas density. In this Letter, we utilize literature data in order to examine the relationship between SFR (traced by the $850 \mu \mathrm{m}$ flux) and dense molecular gas (traced by $\mathrm{CO}(\mathrm{J}=3-2))$. The aim is to help distinguish between the competing interpretations of the linear SFR-dense gas relations in galaxies.

\section{LITERATURE DATA}

In order to investigate the relation between SFR and dense molecular gas over a variety of physical spatial extents in galaxies, we require a beam matched set of observations in both high critical density molecular line and SFR tracer with a sufficient number of galaxies. While matching the highest resolution and sensitivity infrared data of nearby galaxies (e.g SINGS; Kennicutt \& et 2003) with high spatial resolution observations of a high critical density tracer (e.g. Krips et al. 2007) would be ideal, few galaxies exist at the intersection of such surveys. Most other traditional infrared surveys are insufficient as they typically report global measurements, rather than higher spatial resolution data. Further complications arise on the dense gas tracer side. Because of the prodigious observing time necessary to map large numbers of galaxies with an interferometer, most dense gas surveys are done with a single dish and report on lines at $\sim \mathrm{mm}$ wavelengths (e.g. Baan et al. 2008). In order to obtain the highest spatial resolution possible from single dish surveys, higher-frequency observations (e.g. $\lambda<1 \mathrm{~mm}$ ) must be employed.

The largest beam-matched sample of a dense gas tracer and $\mathrm{L}_{\mathrm{IR}}$ tracer is the JCMT CO (J=3-2) survey of Yao et al. (2003) and the SCUBA Local Universe Galaxy Survey (Dunne et al. 2000). Yao et al. (2003) used the $850 \mu \mathrm{m}$ images of these galaxies to scale the total $\mathrm{L}_{\mathrm{IR}}$ to match the $\sim 15^{\prime \prime}$ beam of the $\mathrm{CO}(\mathrm{J}=3-2)$ observations. As such, while the number statistics are relatively small, to our knowledge this sample comprises the largest available for this type of analysis. Moreover, using $\mathrm{CO}(\mathrm{J}=3-2)$ as a tracer of dense molecular gas has the attractive quality that both global measurements (Narayanan et al. 2005) and simulated unresolved observations from simulations (Narayanan et al. 2007) have shown a linear relation between $\mathrm{L}_{\mathrm{IR}}$ and $\mathrm{CO}(\mathrm{J}=3-2)$ luminosity in galaxies.

In order to avoid single galaxies at the extrema of the $\mathrm{L}_{\mathrm{IR}}$ range artificially biasing the fits, we impose nominal luminosity cuts in the sample, considering galaxies in the range $\mathrm{L}_{\mathrm{IR}}=\left[10^{9}, 2 \times 10^{11} \mathrm{~L}_{\odot}\right]$. This excludes galaxies which may be interacting, thus allowing us to use galaxy distance as a proxy for mean density $(\$ 3$ ). This results in a total sample size of 40 galaxies, excluding only 4 galaxies from the parent sample.
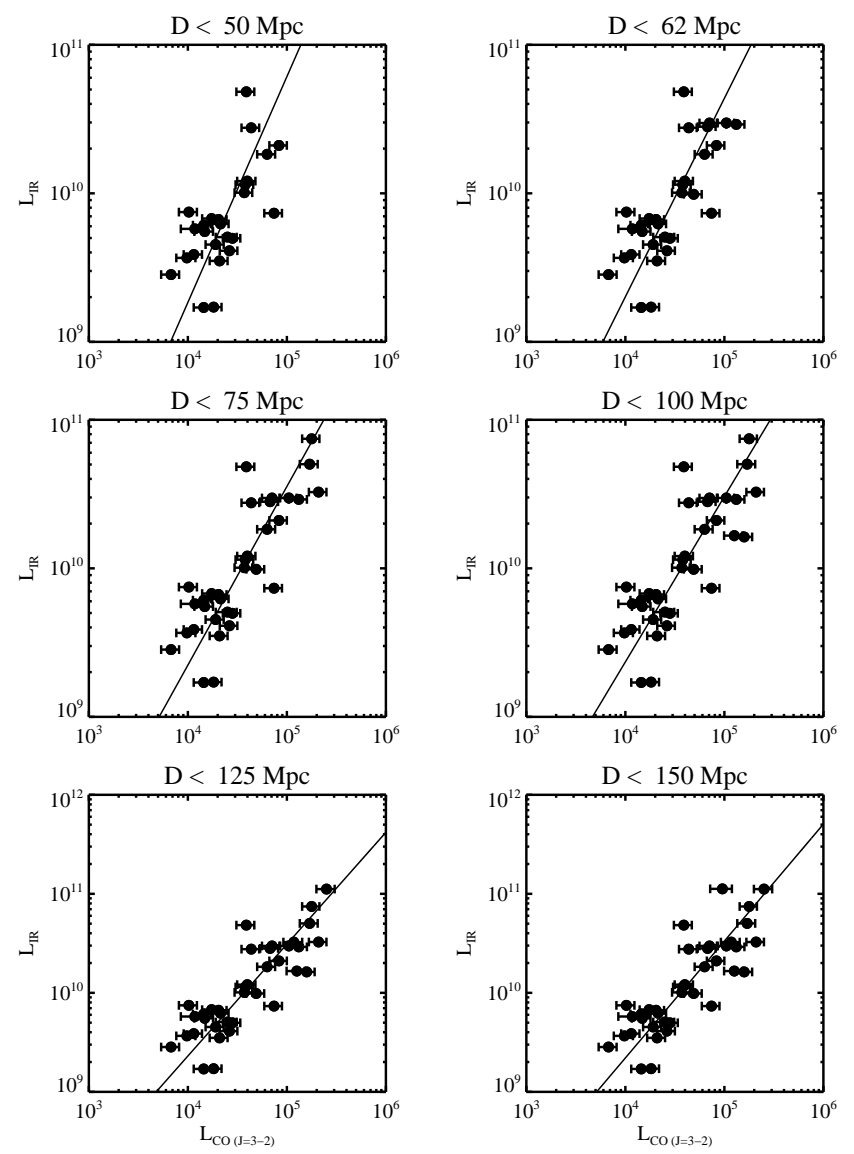

FIG. 1. - The $\mathrm{L}_{\mathrm{IR}}-\mathrm{CO}(\mathrm{J}=3-2)$ relation for galaxies in each of our distance bins. The slope is seen to flatten as observations probe more global measurements.

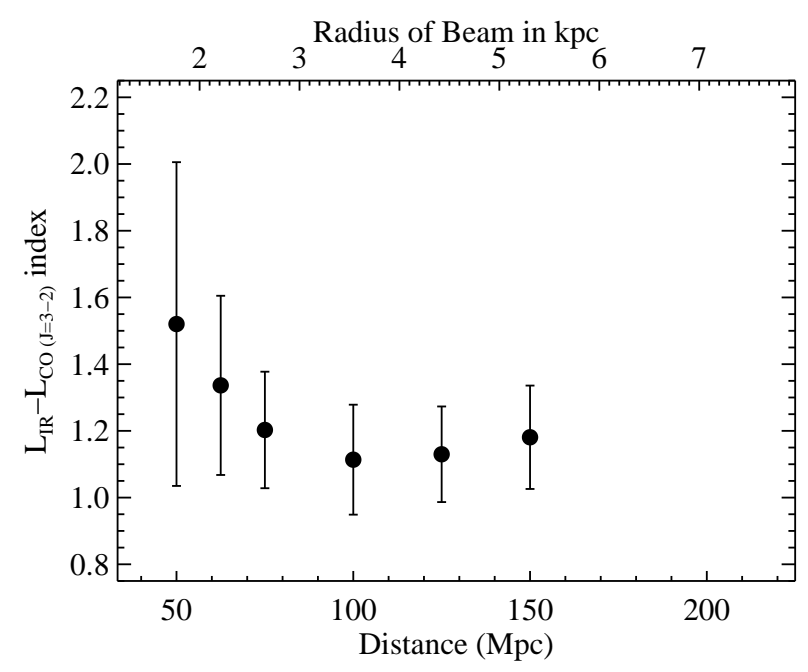

FIG. 2.- The $\mathrm{L}_{\mathrm{IR}}-\mathrm{CO}(\mathrm{J}=3-2)$ index as a function of limiting galaxy distance. The physical scale covered by the beam is on the top axis. Observations of the nearby galaxies probe the nuclear region of the galaxies. In this high $\langle n\rangle$ regime, the $\mathrm{L}_{\mathrm{IR}}-\mathrm{CO}(\mathrm{J}=3-2)$ index tentatively lies near the volumetric Schmidt index, also consistent with recent modeling efforts by Krumholz \& Thompson (2007) and Narayanan et al. (2007). As observations become unresolved and more low density gas is folded into the beam, the index tends toward unity.

\section{RESULTS}

We have binned our sample of galaxies into bins in distance. Because we are considering observations of a fixed beamsize, this is equivalent to binning the galaxies in terms of physi- 
cal radial extent from the nucleus probed by the observations. The number of galaxies rises sharply to $\sim 25$ when considering galaxies with a maximum distance of $\sim 50 \mathrm{Mpc}$. We thus utilize $50 \mathrm{Mpc}$ as the first distance bin in order to maximize the statistical significance of the result.

In Figure 1, we show the $\mathrm{L}_{\mathrm{IR}}-\mathrm{CO}(\mathrm{J}=3-2)$ relation for each distance bin. In Figure 2, we plot the best fit $\log \left(\mathrm{L}_{\mathrm{IR}}\right)$ $\log (\mathrm{CO}(\mathrm{J}=3-2))(\mathrm{L}$; Gao \& Solomon 2004a) slope as a function of limiting distance. On the top axis we label the physical extent of the beamsize. The fits were done utilizing the publically available Monte Carlo Markov Chain fitting packages of Kelly (2007). The Bayesian routines assume the intrinsic distribution can be well approximated via a mixture of Gaussians, and returns the posterior probability distribution function (pdf) of potential slopes to the fit. Unlike standard linear regression techniques, the fit is not done with the assumption that the abscissa values are known exactly while measurement errors exist only for the ordinate. Rather, errors are allowed in both axes. This is important as errors are only reported for the literature CO measurements (Yao et al. 2003), and to conform with standard literature fits, we are required to fit $L_{I R}$ as a function of the CO luminosity. The points in Figure 2 are the median of the returned distribution of slopes, and the error bars denote the standard deviation in the pdf.

The slopes in the $\mathrm{L}_{\mathrm{IR}}-\mathrm{CO}(\mathrm{J}=3-2)$ relation tentatively show a similar trend to what would be expected from the models of Krumholz \& Thompson (2007) and Narayanan et al. (2007). Specifically, in the regions of high $\langle n\rangle$ where the bulk of the gas may be thermalized, the SFR- $\mathrm{L}_{\mathrm{mol}}$ relation for high critical density tracers tends toward 1.5 (e.g. the points at $\mathrm{D} \approx 50 \mathrm{Mpc}$ in Figure 2, this corresponds to the central $\sim 1.7$ $\mathrm{kpc}$ for most of the galaxies in this bin). When considering more unresolved, global observations of galaxies which fold in significant amounts of diffuse gas (thus lowering the effective $\langle n\rangle$ probed by the observations), the mean density of the galaxy drops below the critical density, and the observed SFR- $\mathrm{L}_{\text {mol }}$ index is less than that of the underlying volumetric Schmidt index. Here, we see that the more unresolved observations approach a slope of unity, consistent with the linear slope found between $\mathrm{L}_{\mathrm{IR}}$ and $\mathrm{CO}(\mathrm{J}=3-2)$ luminosity in a nearly identical sample of galaxies by Narayanan et al. (2005). We note that the slope is still moderately superlinear in these bins, though consistent with the range of results expected by the Narayanan et al. (2007) models. This occurs because the maximum distance bins still contain the relatively nearby $(\mathrm{D}<50 \mathrm{Mpc})$ galaxies, which increases the $\mathrm{L}_{\mathrm{IR}}-\mathrm{CO}(\mathrm{J}=3-2)$ index. Ideally one would like to exclude the nearby galaxies from the most distant bins, but low number statistics prevent this experiment.

\section{DISCUSSION}

The trends seen in Figure 2 may be consistent with a picture in which the observed SFR- $\mathrm{L}_{\text {mol }}$ relations are driven globally by the relationship between $\left\langle n_{\text {gas }}\right\rangle$ and $n_{\text {crit }}$. In contrast, if the linear relation between $\mathrm{L}_{\mathrm{IR}}$ and $\mathrm{HCN}(\mathrm{J}=1-0)$ or $\mathrm{CO}(\mathrm{J}=3-2)$ were indicative of a more fundamental SFR relation in galaxies in terms of dense molecular gas, one would expect the relation to remain linear even in the nuclei of galaxies. With a potentially model distinguishing result such as this one, obvious questions regarding its robustness arise.

First, we caution that the error bars presented in the lowest distance bins in Figure 2 are rather large. This owes to the small sample sizes of these bins ( $\sim 25$ galaxies for the lowest distance bins). It is possible to investigate what the probability is that the same $\mathrm{L}_{\mathrm{IR}}-\mathrm{CO}(\mathrm{J}=3-2)$ index properly characterizes both the lowest and highest distance bins. To do this, we remind the reader that each point (and associated error bars) in Figure 2 is a pdf for potential $\mathrm{L}_{\mathrm{IR}}-\mathrm{CO}(\mathrm{J}=3-2)$ indices at each distance bin. In Figure 3, we plot the (normalized) difference in the pdf's from the highest and lowest distance bins in Figure 2. We additionally show the cumulative distribution function in the same plot. A value of 0 in the pdf difference is expected at points when the same index characterizes both the high and low distance bins. As can be seen, the nuclear $\mathrm{L}_{\mathrm{IR}}-\mathrm{CO}(\mathrm{J}=3-2)$ index is systematically weighted toward larger numbers than the global value. That said, the error bars in the nuclear distance bin are not insignificant. The probability that the $\mathrm{L}_{\mathrm{IR}}-\mathrm{CO}(\mathrm{J}=3-2)$ indices from the nuclear and global distance bins are the same $\pm 0.25(0.5)$ is $\sim 17(33) \%$. Thus, while the tentative trends seen in Figure 2 are indeed probable, they are by no means robust. In this sense, surveys to increase the number statistics will be required to confirm/refute this potential result.

Second, we can inquire as to the validity of $\mathrm{L}_{\mathrm{IR}}$ as an SFR indicator. In particular, the $\mathrm{L}_{\mathrm{IR}}$ from galaxies has the potential to be contaminated by central AGN. That said, the galaxies in the sample presented here are relatively low luminosity (global $\mathrm{L}_{\mathrm{IR}}<10^{12} \mathrm{~L}_{\odot}$ ). Both observational studies (Tran \& et 2001) and theoretical works (Chakrabarti et al. 2007) suggest that galaxies with the $\mathrm{L}_{\mathrm{IR}}$ range investigated here have their IR luminosity largely powered by star formation.

Third, we can question whether or not the trend in the $\mathrm{L}_{\mathrm{IR}}{ }^{-}$ $\mathrm{CO}(\mathrm{J}=3-2)$ index seen with distance is equivalent to a trend in $\langle n\rangle$. To do this, in Figure 4, we plot the normalized $\langle n\rangle$ of the galaxy versus the galaxy distance. For the density determination, the volume is derived by assuming the gas resides in a disk with radius equivalent to the physical extent probed by the beam at that distance, and a constant scale height in each galaxy. The molecular gas mass is taken from the $\mathrm{CO}$ $(\mathrm{J}=1-0)$ luminosity, utilizing a $\mathrm{CO}-\mathrm{H}_{2}$ conversion factor appropriate for this sample of galaxies (Yao et al. 2003). The $\mathrm{CO}(\mathrm{J}=1-0)$ observations are beam matched to the $\mathrm{CO}(\mathrm{J}=3-2)$ observations presented here, so the molecular gas mass is indeed the gas mass within the radial extent of the $\mathrm{CO}(\mathrm{J}=3-2)$ beam. The mean density is normalized to account for a variety of uncertain parameters (e.g. disk scale height, molecular gas volume filling factor, density profile of GMCs). Because we are only concerned with the trend in $\left\langle n_{\text {galaxy }}\right\rangle$ with distance, normalizing the density has no consequence. Figure 4 shows that the mean density probed by the molecular line observations drops as we consider galaxies successively farther away. Alternatively said, unresolved observations of galaxies probe lower $\langle n\rangle$ than observations toward galactic nuclei. This implies that the tentative trend in $\mathrm{L}_{\mathrm{IR}}-\mathrm{CO}(\mathrm{J}=3-2)$ index seen in Figure 2 owes to the relationship between the mean density of the gas in the beam and $n_{\text {crit }}$ of the emission line.

If larger samples verify the results of Figure 2, then these observed trends may have several implications. The models of Krumholz \& Thompson (2007) and Narayanan et al. (2007) which predict an SFR- $\mathrm{L}_{\text {mol }}$ index of 1.5 when $\langle n\rangle$ is high are predicated on an underlying relation controlling the SFR: SFR $\propto n^{1.5}$. The results presented here tentatively support a scenario in which the SFR can be described by such a powerlaw (rather than a linear relation), though we reiterate that the error bars in this study are substantial.

In principle, we could perform the same experiment shown in Figure 2 with the $\mathrm{CO}(\mathrm{J}=1-0)$ transition. Indeed, Nobeyama $45 \mathrm{~m}$ observations of $\mathrm{CO}(\mathrm{J}=1-0)$ in many of the same galax- 


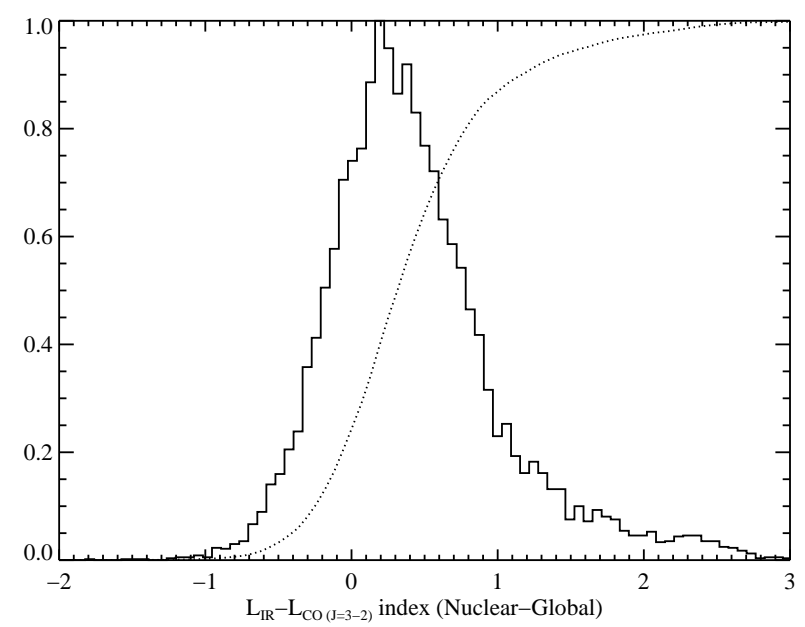

FIG. 3.- Solid Line: Normalized difference in PDFs of $\mathrm{L}_{\mathrm{IR}}-\mathrm{CO}(\mathrm{J}=3-2)$ indices for lowest distance bin and largest distance bin. Dotted line: $\mathrm{Cu}-$ mulative distribution function for shown PDF. This can be interpreted as the $\mathrm{PDF}$ of the $\mathrm{L}_{\mathrm{IR}}-\mathrm{CO}(\mathrm{J}=3-2)$ indices for the nuclear and global observations as being the same.

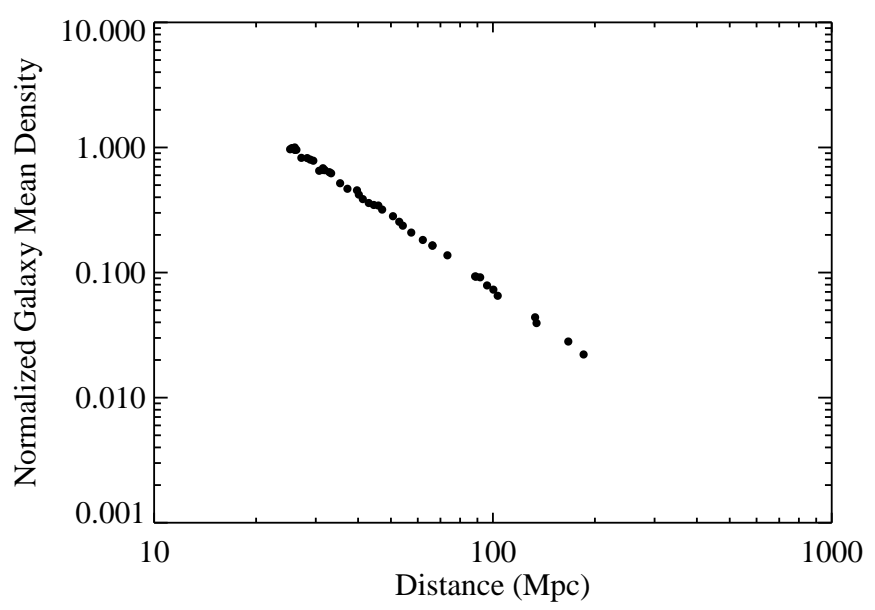

FIG. 4. - The normalized $\langle n\rangle$ within the $\mathrm{CO}(\mathrm{J}=3-2)$ beam as a function of galaxy distance. The $\langle n\rangle$ within the beam drops as a function of distance, showing that the more unresolved observations are probing lower $\langle n\rangle$ gas. This implies that the relationship seen in Figure 2 owes to a dropping $\langle n\rangle$ in the farthest objects.

ies exist, thus providing the opportunity for a beam-matched comparison to this dataset (Yao et al. 2003). However, the error bars on these observations are sufficiently large that the slopes on the $\mathrm{L}_{\mathrm{IR}}-\mathrm{CO}(\mathrm{J}=1-0)$ indices for the lowest distance bins $(\mathrm{D}<100 \mathrm{Mpc})$ are consistent with numerous interpretations (including no correlation at all).

The lack of clean data in low $n_{\text {crit }}$ transitions does not preclude us from conjecturing what results one might expect in a similar experiment with $\mathrm{CO}(\mathrm{J}=1-0)$. Naively, one might imagine the $\mathrm{L}_{\mathrm{IR}}-\mathrm{CO}(\mathrm{J}=1-0)$ index to be $\sim 1.5$ for all distance bins owing to the relatively low critical density of this ground state transition. However, the more likely scenario are results similar to the trend seen in Figure 2 - a slope of $\sim 1.5$ for the lowest distance bins, and a flattening to a slope of $\sim 1$. The reason for this is global observations of galaxies in this relatively low $\mathrm{L}_{\mathrm{IR}}$ range have a linear $\mathrm{L}_{\mathrm{IR}}-\mathrm{CO}(\mathrm{J}=1-0)$ relation (Gao \& Solomon 2004a b). It is only when considering galaxies with $\mathrm{L}_{\mathrm{IR}} \gtrsim 10^{11} \mathrm{~L}_{\odot}$ in the sample that unresolved observations result in an $\mathrm{L}_{\mathrm{IR}}-\mathrm{CO}(\mathrm{J}=1-0)$ index of 1.5. In the interpretation of Krumholz \& Thompson (2007) and Narayanan et al. (2007), this would result from lower luminosity galaxies having most of their gas subthermal in this transition.

Finally, we emphasize that though the results presented here advocate an interpretation of the linear $\mathrm{L}_{\mathrm{IR}}$-dense gas relations consistent with Krumholz \& Thompson (2007) and Narayanan et al. (2007), this is not to say that some high critical density tracers such as $\mathrm{HCN}(\mathrm{J}=1-0)$ are not applicable as SFR tracers. By virtue of their linearity with $\mathrm{L}_{\mathrm{IR}}$, insomuch that the $\mathrm{L}_{\mathrm{IR}}$ can be used as an adequate proxy for the SFR, so can global measurements of $\mathrm{HCN}(\mathrm{J}=1-0)$ and $\mathrm{CO}(\mathrm{J}=3-2)$.

\section{CONCLUSIONS AND WAYS FORWARD}

We have studied the relationship between the SFR and dense molecular content in the nuclei of galaxies by utilizing the observed $\mathrm{L}_{\mathrm{IR}}$ as a proxy for the SFR, and $\mathrm{CO}(\mathrm{J}=3-2)$ as a proxy for dense molecular gas. We find tentative evidence that the $\mathrm{L}_{\mathrm{IR}}-\mathrm{CO}(\mathrm{J}=3-2)$ index is superlinear in galactic nuclei with value $N \sim 1.5$, while flattening as the observations measure more global conditions.

The error bars in this potential trend are considerable however, and that the results here serve as motivation for future observational experiments investigating the $\mathrm{L}_{\mathrm{IR}}$-dense gas relation in a variety of environments. One potential way to increase the sample size would be to obtain additional beammatched sub-mm and $\mathrm{CO}(\mathrm{J}=3-2)$ observations of the nuclei of nearby galaxies with $15 \mathrm{~m}$ sub-mm telescopes (e.g. the JCMT or ASTE). Alternatively, high spatial resolution observations of nearby galaxies with ancillary IR maps (e.g. the SINGS sample), would provide a rich dataset with $\mathrm{L}_{\mathrm{IR}}$ and dense gas measurements for numerous regions with varying physical conditions in individual galaxies. Additional data to constrain the gas density (e.g. $\mathrm{CO} J=1-0$ ) would allow the $\mathrm{L}_{\mathrm{IR}}-\mathrm{CO}(\mathrm{J}=3-2)$ index to be studied truly as a function of gas density, as opposed to galaxy distance. These experiments will help to confirm/refute the potential results of Figure 2 .

We thank Lihong Yao and Brandon Kelly for helpful conversations. Support for this work was provided in part by the Keck Foundation.

\section{REFERENCES}

Baan, W. A., Henkel, C., Loenen, A. F., Baudry, A., \& Wiklind, T. 2008, A\&A, 477, 747

Chakrabarti, S., Cox, T. J., Hernquist, L., Hopkins, P. F., Robertson, B., \& Di Matteo, T. 2007, ApJ, 658, 840

Dunne, L., Eales, S., Edmunds, M., Ivison, R., Alexander, P., \& Clements, D. L. 2000, MNRAS, 315, 115

Gao, Y., Carilli, C. L., Solomon, P. M., \& Vanden Bout, P. A. 2007, ApJ, 660, L93

Gao, Y. \& Solomon, P. M. 2004a, ApJS, 152, 63
-. 2004b, ApJ, 606, 271

Kelly, B. C. 2007, ApJ, 665, 1489

Kennicutt, Jr., R. C. 1998, ARA\&A, 36, 189

Kennicutt, Jr., R. C. \& et, a. 2003, PASP, 115, 928

Krips, M., Neri, R., Garcia-Burillo, S., Martin, S., Combes, F., Gracia-Carpio, J., \& Eckart, A. 2007, ArXiv e-prints, 712

Krumholz, M. R. \& Thompson, T. A. 2007, ApJ, 669, 289

Narayanan, D., Cox, T. J., Shirley, Y., Dave, R., Hernquist, L., \& Walker, C. K. 2007, ArXiv e-prints, 711 
Narayanan, D., Groppi, C. E., Kulesa, C. A., \& Walker, C. K. 2005, ApJ, 630, Yao, L., Seaquist, E. R., Kuno, N., \& Dunne, L. 2003, ApJ, 588, 771 269

Schmidt, M. 1959, ApJ, 129, 243

Tran, Q. D. \& et, a. 2001, ApJ, 552, 527

Wu, J., Evans, II, N. J., Gao, Y., Solomon, P. M., Shirley, Y. L., \& Vanden Bout, P. A. 2005, ApJ, 635, L173 